Reply

\title{
The threshold of wakefulness, the experience of control, and theory development ${ }^{\text {s }}$
}

\author{
Timothy Lane ${ }^{\mathrm{a}}$, Chien-Ming Yang a,b,* \\ ${ }^{a}$ The Research Center for Mind, Brain, and Learning, National Chengchi University, Taipei, Taiwan \\ ${ }^{\mathrm{b}}$ Department of Psychology, National Chengchi University, Taipei, Taiwan
}

\section{A R T I C L E I N F O}

\section{Keywords:}

Sleep onset

Control

Subjective experience

Perception of sleep

We are very grateful to Professor Wackermann for his constructive and insightful comments. We take it that one among his main concerns is the possibility of variation that might go undetected due to our choice of methodology. For example, when inquiring as to the logicality or coherence of thoughts, we seem to be presupposing a shared internal norm that can be accurately reflected, despite multiple, mediating steps of memory and evaluation.

Professor Wackermann's concern is by no means an idle one. But (1) we believe that queries of the sort employed here are necessary if we are to begin making progress in eliciting the structure of conscious experience. Were we to limit ourselves to questions that concern just raw, sensory experience, we would risk arbitrarily ignoring significant aspects of the subjects' phenomenology. Further, (2) our statistical analysis is indeed intended to balance out individual differences. Although this strategy does risk obscuring important variation, it can still be helpful in identifying significant, albeit not universal, indicators. Finally, (3) while it is possible that the transition from wakefulness to sleep follows different paths, the issue is an empirical one. Just as it would be unwise to arbitrarily ignore individual variation, so too would it be unwise to arbitrarily inflate individual variation.

We realize that Professor Wackermann's concerns though are not mere methodological quibbles as regards how best to address a single psychological phenomenon. As Wackermann (2006) lucidly expresses elsewhere, he seeks to develop a strategy for discovering universal laws that is compatible with the study of entities that exhibit great variation, human beings. Indeed, we are in sympathy with his view that more attention should be given to what he terms the "idiomatic" regularities. On this view, research should proceed in a two-step fashion: first, one should attend to intra-individual regularities and render these in logical or mathematical form. Only after this step has been completed should one seek inter-individual comparisons.

As regards the research that actuated Professor Wackermann's critique, we are not able to present results in such a way that they would satisfy strict standards for "distributed nomothesis." But, motivated by this strategy, we have re-evaluated the data, attending more carefully to individual variation. In so doing we found that, for most subjects, ratings on more than one item were associated with the perception of falling asleep. Moreover, for ten of the twenty subjects, "control over think-

\footnotetext{
Reply to Commentary by Wackermann on Yang, C.-M., Han, H.Y., Yang, M.H., Su, W.C., \& Lane, T. (2010). What subjective experiences determine the perception of falling asleep during sleep onset period? Consciousness and Cognition 19, 1084-1092.

* Corresponding author. Address: Department of Psychology, National Chengchi University, 64, Sec. 2, Chih-Nan Rd., Taipei 116, Taiwan.

E-mail address: dryangcm@gmail.com (C.-M. Yang).
} 
ing process" was associated with the perception of falling asleep; for eight subjects, "control over perception"; and, for seven, "thinking experience," "logic of thinking process," and "orientation."

This reanalysis suggests that the experience of control might be a key factor in the subjective experience of sleep onset. Not only is it cited explicitly with reference to thought process and perception, it seems to be implied by those who indicate that their thoughts were not logical. Speculating, perhaps further investigations of the relevant cognitive processes would reveal that one among the significant idiomatic regularities related to this transitional state involves diminution of the sense of control.

Presumably the relevant meaning of control here does not concern the obsessive or intrusive thoughts that are commonly associated with insomnia-after all, the reported experiences are regarded by the subjects as indications of sleep onset. It would seem to be far more likely that the relevant sense of control bears greater similarity to the thought insertions experienced by schizophrenics, what Frith (1992) refers to as "passivity experiences." Frith's account might also help to explain the association with "control over perception," as his model emphasizes our capacity to distinguish between changes in our perception of the external world that result from our own actions and changes that result from alterations in the external environment itself. In schizophrenics this ability is impaired, an impairment that Frith attributes to a failure to monitor intentions. Inability to monitor intentions might well be experienced as an inability to control perception.

Naturally we do not intend to suggest that sleep onset and schizophrenia are one and the same. Clearly the two differ in many respects. But exploration of the nature and degree of difference might well lead to significant insights.

Such exploratory work would, we believe, be consistent with Wackermann's (2006) view that science should be dedicated to the search for a "beautiful linking of facts." He believes that too much experimental work is nothing more than "a game played by its own rules on an isolated playground." He advocates regarding experimental work as "materialized reasoning": that is, experiments should be motivated by careful theory development that is relatively independent of particular databases. One goal of such development should be a "beautiful linking of facts," where previously there had only been a disconnected jumble.

As a very preliminary step in the direction of finding pattern amid jumble, recent research into control of action and goal maintenance suggests a separate, but arguably relevant domain. For example, Suhler and Churchland (2009) have proposed a neurobiological model of control that is applicable both to quotidian states wherein control is exercised (e.g. getting out of a warm bed on a cold morning) and to prototypical cases wherein persons feel "out of control" (e.g. addiction). They propose a model of multiple parameters that includes neurochemicals, connectivity among brain structures, and so forth. The intent is to identify an "in control" region within multi-dimensional space, a space that reflects the likelihood that there are many different ways of being in, or out, of control.

Were we to further develop such a model, because the prefrontal cortex is implicated in self-monitoring and in goaldirected thought and because it is relatively inactive during NREM sleep (Muzur, Pace-Schott, \& Hobson, 2002), we would likely include it as one among several parameters that needs to be highlighted. Nevertheless, we are keenly aware that our brief discussion here merely gestures in the direction of one possible line of inquiry. But an especially attractive feature of such theorizing is that it allows for the possibility of mathematical modeling in a way that can accommodate "idiomatic" regularities: that is, it can account for different ways of being in and out of control. Of course whether or not thinking along the lines adumbrated here will yield fruitful results, we cannot say. But we are grateful for Professor Wackermann's gentle encouragement to search for the idiomatic and to take seriously the role of theorizing.

\section{References}

Frith, C. (1992). The cognitive neurobiology of schizophrenia. New Jersey: Lawrence Erlbaum Associates.

Muzur, A., Pace-Schott, E. F., \& Hobson, J. A. (2002). The prefrontal cortex in sleep. Trends in Cognitive Sciences, 6, 475-481.

Suhler, C., \& Churchland, P. S. (2009). Control: Conscious and otherwise. Trends in Cognitive Sciences, 13, 341-347.

Wackermann, J. (2006). Rationality, universality, and individuality in a functional conception of theory. International Journal of Psychophysiology, 62, 411-426.

Yang, C.-M., Han, H. Y., Yang, M. H., Su, W. C., \& Lane, T. (2010). What subjective experiences determine the perception of falling asleep during sleep onset period? Consciousness and Cognition, 19, 1084-1092. 